\title{
Pacientes assintomáticos apresentam infecção relacionada ao cateter venoso utilizado para terapia nutricional parenteral
}

\author{
Asymptomatic patients present infection related \\ to the central venous catheter used \\ for total parenteral nutrition
}

Juliana Deh Carvalho MACHADO'

Vivian Marques Miguel SUEN²

José Fernando de Castro FIGUEIREDO ${ }^{3}$ (in memorian)

Júlio Sérgio MARCHINI ${ }^{3}$

RE S U M O

\section{Objetivo}

Avaliar a freqüência de infecção relacionada ao cateter venoso central em pacientes submetidos a terapia nutricional parenteral.

\section{Métodos}

Foram analisados os cateteres venosos centrais de pacientes em terapia nutricional parenteral que tiveram a indicação de retirada do cateter venoso central por infecção, alta hospitalar, ou trombose. Os pacientes com infecção foram denominados de Grupo 1 e os demais de Grupo 2.

\section{Resultados}

Não houve diferença estatisticamente significante quanto ao estado nutricional dos 18 pacientes analisados. Foram analisados 28 cateteres e destes 68\% estavam infectados, sendo $72 \%$ do Grupo 1 e $28 \%$ do Grupo 2 (assintomáticos). No Grupo 1, houve infecção sistêmica em 70\% dos casos, já no Grupo 2 a hemocultura foi positiva em $17 \%$ dos casos. A colonização por Staphylococcus sp. ocorreu em $48 \%$ dos casos, seguida de Candida sp. (21\%), Enterococcus faecalis (16\%), Pseudomonas aerurginosa (10\%) e Proteus sp. (5\%).

1 Universidade de São Paulo, Faculdade de Medicina de Ribeirão Preto, Departamento de Clínica Médica, Hospital das Clínicas. Av. Bandeirantes, 3900, 14048-900, Ribeirão Preto, SP, Brasil. Correspondência para/Correspondence to: J.D.C. MACHADO. E-mail: <juliana@infonet.com.br>.

2 Universidade de São Paulo, Faculdade de Medicina de Ribeirão Preto, Departamento de Clínica Médica do Hospital das Clínicas, Divisão de Nutrologia. Ribeirão Preto, SP, Brasil.

3 Universidade de São Paulo, Faculdade de Medicina de Ribeirão Preto, Departamento de Clínica Médica do Hospital das Clínicas, Divisão Moléstias Infecciosas. Ribeirão Preto, SP, Brasil. 
788 | J.D.C. MACHADO et al.

\section{Conclusão}

A contaminação de cateter venoso central utilizado para terapia nutricional parenteral é freqüente. Mesmo pacientes assintomáticos recebendo nutrição parenteral têm uma incidência maior de infecção por Candida sp. Portanto é necessária a criação de barreiras que impeçam a colonização destes cateteres venosos centrais, a fim de diminuir a morbimortalidade de pacientes dependentes deste tipo de terapia.

Termos de indexação: Infecção. Infecções relacionadas a cateter. Nutrição parenteral total.

\section{A B S T R A C T}

\section{Objective}

The aim of this study was to evaluate the frequency of central venous catheter-related infections in hospitalized patients receiving total parenteral nutrition.

\section{Methods}

Central venous catheters were analyzed immediately after removal due to infection, hospital discharge or thrombosis. The patients with catheter-related infection were named Group 1 and the other patients were named Group 2.

\section{Results}

Eighteen patients were studied. There was no statistically significant difference in nutritional status between the two groups. A total of 28 catheters were analyzed. Sixty-eight percent of the catheters were infected: $72 \%$ of them were from Group 1 and 28\% from Group 2 (asymptomatic patients). Systemic infection was diagnosed in $70 \%$ of the patients from Group 1. Positive blood culture was found in 17\% of the patients from Group 2. The microorganisms found were: Staphylococcus sp. (48\%), Candida sp. (21\%), Enterococcus faecalis (16\%), Pseudomonas aerurginosa (10\%) and Proteus sp. (5\%).

\section{Conclusion}

Central venous catheter infection is common in hospitalized asymptomatic patients. Patients receiving total parenteral nutrition are most frequently infected with Candida sp. Therefore, the creation of barriers that block colonization in the central venous catheter is essential to decrease the morbidity and mortality among patients that depend on total parenteral nutrition.

Indexing terms: Infection. Catheter-related infections. Parenteral nutrition total.

\section{N T R O D U Ç Ã O}

Em 1968, Dudrick et al. ${ }^{1}$ relataram, em estudo experimental, a curva de crescimento normal em cachorros alimentados exclusivamente por nutrição parenteral. Ainda nesta década, Shils et $a^{2}$. conseguiram acompanhar pacientes com necessidade de terapia nutricional parenteral domiciliar. A partir desta época a mortalidade de pacientes sem trato disgetivo funcionante diminuiu drasticamente ${ }^{3}$.

A indicação para terapia nutricional parenteral ocorre quando pacientes possuem falência intestinal, seja decorrente de processos obstrutivos, de não motilidade, de ressecções intestinais intensas, defeitos congênitos e doenças que comprometem a absorção, caracterizadas pela incapa- cidade de manter o equilíbrio de energia, proteína, hidroeletrolítico e de micronutrientes ${ }^{4}$. Porém, como todo tratamento clínico, a nutrição parenteral não está isenta de complicações clínicas e a infecção relacionada com cateter venoso central é uma das mais temidas e freqüentes ${ }^{5}$.

O objetivo deste estudo é comparar a incidência de infecção relacionada ao cateter venoso central em pacientes com e sem clínica de infecção, que estavam sendo submetidos à terapia nutricional parenteral.

\section{M É TODOS}

Este foi um estudo prospectivo de cateteres venosos centrais retirados de pacientes internados 
no Hospital das Clínicas da Faculdade de Medicina de Ribeirão Preto da Universidade de São Paulo. Todos os cateteres foram retirados por indicação médica, tais como complicação infecciosa ou mecânica, ou pelo término da terapia que indicou sua utilização. Os pesquisadores responsáveis pela pesquisa não tiveram qualquer participação na indicação da terapia, na evolução do paciente ou na indicação da retirada do cateter. O projeto foi aprovado pelo Comitê de Ética do Hospital das Clínicas da Faculdade de Medicina de Ribeirão Preto (processo HCRP n 13050/2006).

Participaram do estudo pacientes do Hospital das Clínicas da Faculdade de Medicina de Ribeirão Preto que necessitaram de acesso venoso central para terapia nutricional parenteral, em algumas situações os cateteres de duplo lúmen também foram utilizados para administração de medicamentos.

Todos os pacientes que tiveram indicação da retirada do cateter venoso central foram acompanhados e avaliados clinicamente, a fim de diagnosticar sinais clínicos que sugerissem infecção como: hiperemia no local de inserção do cateter, secreção purulenta pericateter, taquicardia, febre, calafrios, bacteremia e sepse sem foco infeccioso conhecido, neste caso o processo infeccioso foi atribuído ao cateter.

Dessa maneira, foram avaliados cateteres venosos centrais utilizados para nutrição parenteral, que foram retirados dos pacientes por indicação médica: seja por infecção (Grupo 1), ou então por não ter mais indicação clínica para sua utilização, ou seja, pacientes que receberam alta médica, ou então tiveram complicação mecânica relacionada ao cateter, como trombose, neste último grupo durante a evolução não foi clinicamente diagnosticada infecção (Grupo 2).

As pontas dos cateteres foram submetidas à cultura semiquantitativa utilizando técnicas classicamente descritas, sendo considerado o teste positivo quando o resultado do método semi- quantitativo fosse $\geq 15$ Unidades Formadoras de Colônia (UFC).

Foi realizada uma análise descritiva. Utilizou-se o teste $t$ de Student para comparar médias populacionais, além do teste exato de Fisher. Em todas as situações, considerou-se um nível de significância estatística de 5\%. Os resultados foram considerados duvidosos quando o $p$ valor foi menor que 0,10 ou maior do que 0,05 .

\section{RESULTADOS}

Foram analisados 18 pacientes submetidos à Terapia Nutricional Parenteral (TNP), que utilizaram um total de 28 cateteres venosos centrais. Destes 16 pertenciam ao Grupo 1 (clinicamente infectados) e 12 ao Grupo 2 (sem sinal clínico de infecção).

As indicações para TNP e as principais características dos pacientes divididos por grupo estão descritas na Tabela 1, sendo a síndrome do intestino curto $(68 \%)$ a mais freqüente. Embora os pacientes do Grupo 1 fossem mais hipoalbuninêmicos e mais emagrecidos do que os do Grupo 2 , não houve diferença entre o estado nutricional dos pacientes dos dois grupos, quanto ao índice de massa corporal, albumina sérica e glóbulos brancos (Tabela 1). Observou-se que $61 \%$ dos pacientes eram linfopênicos e o Grupo 2 apresentava maior incidência de linfopenia (42\%) do que os pacientes do Grupo 1 (38\%).

A Figura 1 mostra a curva térmica axilar média dos dois grupos. Observou-se que os pacientes com infecção clínica sintomática (Grupo 1) apresentavam temperatura axilar superior à do Grupo 2. A Figura 2 exibe os resultados das temperaturas axilares individualizadas de cada grupo.

As características dos cateteres utilizados e o seu sítio de inserção estão resumidos na Tabela 2. O cateter mais utilizado no Grupo 1 foi o de duplo-lúmen (Arrow International ${ }^{\circledR}$, Estados Unidos da América) com 50\% das cateterizações, No Grupo 2, o mais utilizado foi o totalmente implantável (Port-cath ${ }^{\circledR}$, Arrow International ${ }^{\circledR}$, Estados 
790 | J.D.C. MACHADO et al.

Tabela 1. Características gerais de pacientes submetidos à terapia nutricional parenteral. Ribeirão Preto (SP), 2006.

\begin{tabular}{|c|c|c|c|c|c|}
\hline \multirow[t]{2}{*}{ Características } & \multicolumn{2}{|c|}{$\begin{array}{c}\text { Grupo } 1 \\
\text { (Infecção clínica) }\end{array}$} & \multicolumn{2}{|c|}{$\begin{array}{c}\text { Grupo } 2 \\
\text { (Sem infecção clínica) }\end{array}$} & \multirow[t]{2}{*}{$p$} \\
\hline & $M$ & $D P$ & $M$ & $D P$ & \\
\hline Idade (anos) & 55 & $10^{*}$ & 52 & $20^{*}$ & \\
\hline Sexo & $n$ & $\%$ & $n$ & $\%$ & - \\
\hline Masculino & 9 & 81 & 4 & 57 & \\
\hline \multirow[t]{2}{*}{ Feminino } & 2 & 19 & 3 & 43 & \\
\hline & M & $D P$ & $M$ & $D P$ & - \\
\hline Peso $(\mathrm{kg})$ & 46 & 17 & 47 & 21 & \\
\hline IMC $\left(\mathrm{kg} \cdot \mathrm{m}^{-2}\right)$ & 17 & $7^{*}$ & 19 & $6^{*}$ & 0,01 \\
\hline Albumina $\left(\mathrm{g} \cdot \mathrm{L}^{-1}\right)$ & 28 & $6^{*}$ & 32 & $6^{*}$ & 0,08 \\
\hline Leucócitos $\left(\mathrm{mm}^{-3}\right)$ & 8369 & $4072^{*}$ & 8460 & $5783^{*}$ & 0,48 \\
\hline Linfócitos $\left(\mathrm{mm}^{-3}\right)$ & 1548 & 1342 & 1396 & 1204 & 0,38 \\
\hline Linfopenia n (\%) & 6 & 38 & 5 & 42 & \\
\hline Doenças associadas & $n$ & $\%$ & $n$ & $\%$ & - \\
\hline SIC & 8 & 73 & 4 & 57 & \\
\hline DM & 2 & 18 & - & - & \\
\hline $\mathrm{HA}$ & 6 & 55 & 1 & 14 & \\
\hline DAC & 1 & 9 & 1 & 14 & \\
\hline DPC & 10 & 9 & 5 & 72 & \\
\hline Insuficiência cardíaca & 2 & 18 & 1 & 14 & \\
\hline Diarréia intratável & 1 & 91 & 4 & 57 & \\
\hline Fístula entérica & 1 & 9 & - & - & \\
\hline Oclusão intestinal & 1 & 9 & - & - & \\
\hline Mieloma múltiplo & 1 & 9 & - & - & \\
\hline Esclerose múltipla & - & - & 1 & 14 & \\
\hline Carcinoma & 3 & 28 & 1 & 14 & \\
\hline
\end{tabular}

IMC: índice de massa corporal; SIC: síndrome do intestino curto; HA: hipertensão arterial; DAC: doença aterosclerótica. DPC: desnutrição protéico-energética; DP: desvio-padrão; M: média.

*Média e Desvio-Padrão

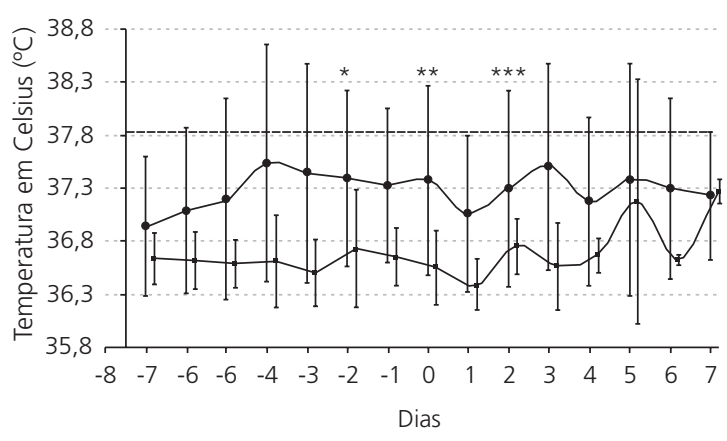

$\rightarrow$ Grupo $1 \rightarrow$ Grupo $2 \quad$---- Hipertemia

Figura 1. Curva térmica axilar média de pacientes submetidos à terapia nutricional parenteral. Ribeirão Preto (SP), 2006.

Notas: ${ }^{*} p<0,005 ;{ }^{* *} p<0,03,{ }^{* * *} p<0,05$.

O Grupo 1 representa pacientes com sintomas clínicos de infecção. O Grupo 2 representa os pacientes sem sintomas e sinais de infecção relacionada ao cateter. A linha pontilhada representa o ponto de corte de hipertermia e febre. Dia 0 representa o dia da retirada do cateter.
Unidos da América), representando $50 \%$ dos casos. A jugular interna foi o local de inserção mais utilizado pela equipe médica (63\%).

A incidência de colonização da ponta dos cateteres do Grupo 1 foi de $81 \%$ e a do Grupo 2 de $50 \%$, porém, dos cateteres que estavam colonizados apenas 56\% da amostra do Grupo 1 tiveram hemoculturas positivas, ou seja tiveram infecção na corrente sangüínea relacionada com o cateter (Tabela 2). Já no Grupo 2, esta relação só ocorreu em $8 \%$ do casos. A taxa de infecção total foi de 0,049 pacientes por ano. No Grupo 1 foi de 0,51 pacientes por ano, enquanto no Grupo 2 foi de 0,015 pacientes por ano.

Os pacientes sem sinais clínicos de infecção permaneceram por mais tempo com o acesso venoso (294, Desvio-Padrão - DP=453 dias), versus 


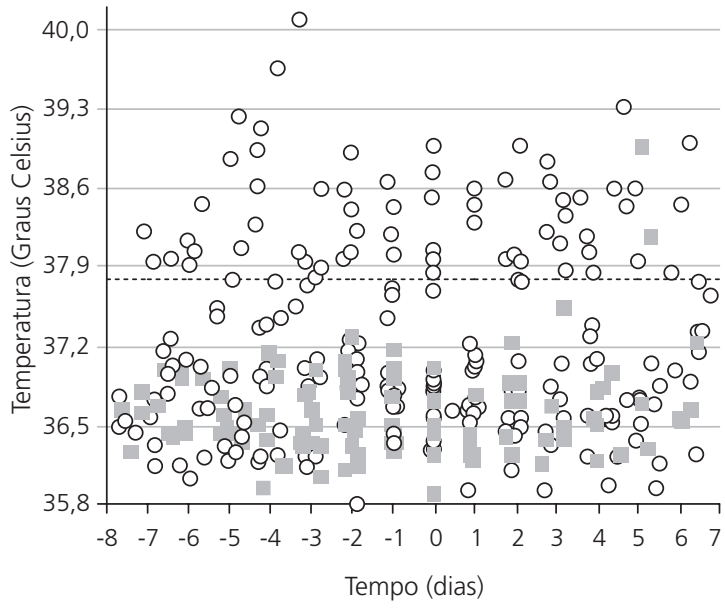

○ Grupo $1 \square$ Grupo 2 ......... Hipertermia

Figura 2. Temperatura axilar de pacientes submetidos à terapia nutricional parenteral. Ribeirão Preto (SP), 2006

Notas: O Grupo 1 representa pacientes com sintomas clínicos de infecção. O Grupo 2 representa os pacientes sem sintomas e sinais de infecção relacionada ao cateter. A linha pontilhada representa o ponto de corte de hipertermia e febre. $\mathrm{O}$ dia 0 (zero) representa o dia da retirada do cateter.

$37(\mathrm{DP}=66)$ dias do grupo com infecção clínica $(p=0,038)$.

Ao serem submetidas à cultura semiquantitativa observou-se que $81 \%$ das pontas analisadas no Grupo 1 foram positivas, enquanto que no Grupo 2 a positividade ocorreu em 50\% dos casos. Os cateteres inseridos na veia jugular foram os que apresentaram a maior freqüência de colonização ou de infecção relacionada ao cateter, sendo esta de $64 \%$, seguida da femoral $50 \%$ e da subclávia $47 \%$.

O staphylococcus sp. foi o microorganismo mais freqüente encontrado nos $C V C$, seguido por: cândidasp., enterococcussp., proteus, pseudomonas e escherichia coli.

\section{I S C U S S Ã O}

Um processo infeccioso nem sempre é diagnosticado clinicamente, alguns pacientes, principalmente os imunodeprimidos, podem evoluir clinicamente sem os sintomas clássicos de
Tabela 2. Características dos cateteres venosos centrais de pacientes submetidos à terapia nutricional parenteral. Ribeirão Preto (SP), 2006.

\begin{tabular}{|c|c|c|c|c|}
\hline \multirow[b]{2}{*}{ Indicação terapêutica } & \multicolumn{2}{|c|}{$\begin{array}{c}\text { Grupo } 1 \\
\text { (Infecção } \\
\text { clínica) }\end{array}$} & \multicolumn{2}{|c|}{$\begin{array}{c}\text { Grupo } 2 \\
\text { (Sem infecção } \\
\text { clínica) }\end{array}$} \\
\hline & $n$ & $\%$ & $n$ & $\%$ \\
\hline Terapia nutricional & 16 & 100 & 12 & 92 \\
\hline Hidratação & 16 & 100 & 12 & 100 \\
\hline Monitorização clínica & - & - & 1 & 8 \\
\hline Tipos & $n$ & $\%$ & $n$ & $\%$ \\
\hline Totalmente implantável & 2 & 13 & 6 & 50 \\
\hline Lúmen simples & 6 & 37 & - & - \\
\hline Duplo-lúmen & 8 & 50 & 5 & 42 \\
\hline Umbilical & - & - & 1 & 8 \\
\hline Tempo de acesso (dias) & $37^{1}$ & $66^{1}$ & $294^{1}$ & $453^{1}$ \\
\hline Motivo da retirada do cateter & $n$ & $\%$ & $n$ & $\%$ \\
\hline Suspensão da terapêutica & - & - & 6 & 50 \\
\hline Bacteremia & 1 & 6 & - & - \\
\hline Febre & 13 & 81 & - & - \\
\hline Hiperemia local inserção & 2 & 13 & - & - \\
\hline Trombose & - & - & 6 & 50 \\
\hline Cultura da ponta & $n$ & $\%$ & $n$ & $\%$ \\
\hline Positiva & 13 & $81^{2}$ & 6 & $50^{2}$ \\
\hline Negativa & 3 & 19 & 6 & 50 \\
\hline Hemocultura ${ }^{3}$ & $n$ & $\%$ & $n$ & $\%$ \\
\hline Positiva & 9 & $69^{2}$ & 1 & $17^{2}$ \\
\hline Negativa & 13 & 31 & 5 & 83 \\
\hline
\end{tabular}

${ }^{1} \mathrm{M}$ : média; DP: desvio-padrão; ${ }^{2} \chi^{2}: 0,09 ;{ }^{3}$ incluem somente pacientes com cultura da ponta positiva.

infecção, fato que pode dificultar o diagnóstico precoce e o tratamento do processo infeccioso, além de aumentar a morbimortalidade desta população. A subnutrição produz alterações morfológicas em, virtualmente, todos os órgãos. A gravidade da disfunção depende, em parte, do gasto energético e da taxa de renovação celular de determinado tecido ou órgão. Neste aspecto, o sistema imune e o gastrintestinal são os mais vulneráveis ${ }^{6}$. A maioria dos pacientes em TNP avaliados era desnutrida e a maioria era linfopênica, fato que pode sugerir imunodepressão e justificar que, mesmos pacientes assintomáticos cursassem com colonização do cateter, ou infecção sistêmica relacionada ao cateter. Neste estudo a linfopenia foi mais freqüente nos pacientes do Grupo 2. 
A fim de combater a desnutrição em pacientes com falência intestinal, ou naqueles em que há contra-indicação de utilizar o trato gastrintestinal, a TNP está indicada, sendo necessário acesso venoso para iniciar a terapia nutricional ${ }^{4}$. Porém, apesar de indispensável para manutenção da vida da maioria de pacientes com insuficiência intestinal, a TNP não está isenta de complicações clínicas, sendo a infecção relacionada ao cateter venoso freqüente ${ }^{5}$. Em estudo com pacientes que recebiam Nutrição Parenteral Prolongada (NPP) por cateteres de longa permanência, Unamuno et al. ${ }^{7}$, em estudo em Ribeirão Preto, obtiveram uma taxa relacionada ao cateter inferior à deste estudo, de 0,029 paciente. Colomb et al. ${ }^{8}$, em um hospital pediátrico francês, obtiveram taxas de 0,78 por paciente ano em população que também necessitava de NPP.

Vários são os fatores relacionados às infecções de CVC dentre os quais citam-se a migração de microorganismos da pele do paciente para o lúmen do cateter ${ }^{9}$, a manipulação do CVC para administração de medicamentos ${ }^{10}$, as condições clínicas dos pacientes que necessitam do acesso central, como idade, ${ }^{11}$ e o uso de nutrição parenteral. Tal fator de risco foi confirmado por Beghetto et al..$^{12}$, em estudo de coorte realizado no Hospital das Clínicas de Porto Alegre durante o período de um ano, comparando o fator de exposição (administração de nutrição parenteral) em pacientes com CVC, no qual os autores observaram um risco relativo de 3,30, com intervalo de confiança de 1,3-8,3 da população exposta comparada à não exposta. Ainda neste estudo, a desnutrição, o tempo de internação que antecedeu a inserção do CVC e a hiperglicemia sustentada não foram fatores de risco para aumento de infecção ${ }^{12}$.

Quanto à epidemiologia dos microorganismos mais freqüentes nesta população, observou-se que a maioria dos cateteres foi colonizada por Staphylococcus coagulase negativo (24\%) e Staphylococcus aureus (24\%), que, somados perfazem, $48 \%$ dos casos, semelhantes a resultados do Centers for Disease Control and Prevention ${ }^{11}$, que apresentam taxas de $50 \%$ de colonização de CVC por estes microorganismos.

\section{O N CLUS ÃO}

A infecção relacionada ao CVC em pacientes submetidos à terapia nutricional parenteral é freqüente, mesmo em população assintomática, sendo necessário cuidado ao manipular estes cateteres venosos centrais, além de desenvolver técnicas de assepsia eficientes que bloqueiem ou impeçam a colonização destes dispositivos médicos.

\section{REFERÊ NCIAS}

1. Dudrick SJ, Wilmore DW, Vars HM, Rhoads JE. Long-term total parenteral nutrition with growth, development, and positive nitrogen balance. Surgery. 1968; 64(1):134-42.

2. Shils $M E$, Wright $W L$, Turnbull $A$, Brescia F. Long term parenteral nutrition through external arteriovenous shunt. N Engl J Med. 1970; 283(7): 341-4.

3. Ireton-Jones C, DeLegge M. Home parenteral nutrition registry: a five-year retrospective evaluation of outcomes of patients receiving home parenteral nutrition support. Nutrition. 2005; 21(2):156-60. doi:10.1016/j.nut.2004.04.024.

4. O'Keefe SJ, Buchman A, Fishbein T, Jeejeebhoy K, Jeppesen P, Shaffer J. Short bowel syndrome and intestinal failure: consensus definitions and overview. Clin Gastroenterol Hepatol. 2006; 4(1): 6-10. doi:10.1016/j.cgh.2005.10.002.

5. O'Keefe SJ, Burnes JU, Thompson RL. Recurrent sepsis in home parenteral nutrition patients: an analysis of risk factors. J Parenter Enteral Nutr. 1994; 18(3): 256-63. doi: 10.1177/01486071940 18003256

6. Caballero B. Global patterns of child health: the role of nutrition. Ann Nutr Metab. 2002; 46(1): S3-S7. doi: 10.1159/000066400.

7. Unamuno MRDL, Carneiro JJ, Chueire FB, Marchini $J S$, Suen VMM. Uso de cateteres venosos totalmente implantados para nutrição parenteral: cuidados, tempo de permanência e ocorrência de complicações infecciosas. Rev Nutr. 2005; 18(2): 261-9. doi: 10.1590/\$1415-527320050002000 10. 
8. Colomb V, Fabeiro M, Dabbas M, Goulet O, Merckx J, Ricour C. Central venous catheter-related infections in children on long-term home parenteral nutrition: incidence and risk factors. Clin Nutr. 2000; 19(5):355-9. doi: 10.1054/clnu.2000. 0132.

9. Veenstra DL, Saint S, Saha S, Lumley T, Sullivan SD. Efficacy of antiseptic-impregnated central venous catheter in preventing catheter-related bloodstream infection: a meta-analysis. JAMA. 1999; 281(3):261-7.

10. Sherertz RJ. Update on vascular catheter infections. Curr Opin Infect Dis. 2004; 17(4):303-7. doi: 10.1097/01.qco.0000136925.52673.16.
11. Centers for Disease Control and Prevention. Guidelines for the prevention of intravascular catheter-related infections. MMWR. 2002; 51(10): $1-34$.

12. Beghetto MG, Victorino J, Teixeira L, Azevedo MJ. Parenteral nutrition as a risk factor for central venous catheter: related infection. J Parenter Enteral Nutr. 2005; 29(5):367-73. doi: 10.1177/014860 7105029005367.

Recebido em: 5/12/2007

Versão final reapresentada em: 22/8/2008 Aprovado em: 9/9/2009 